\title{
Analysis of Filtering Methods for Satellite Autonomous Orbit Determination Using Celestial and Geomagnetic Measurement
}

\author{
Xiaolin Ning, ${ }^{1,2,3}$ Xin Ma, 1,2,3 Cong Peng, ${ }^{1,2,3}$ \\ Wei Quan, ${ }^{1,2,3}$ and Jiancheng Fang ${ }^{1,2,3}$ \\ ${ }^{1}$ School of Instrumentation Science E Opto-electronics Engineering, Bei Hang University (BUAA), \\ Beijing 100191, China \\ ${ }^{2}$ Science and Technology on Inertial Laboratory, Beijing 100191, China \\ ${ }^{3}$ Fundamental Science on Novel Inertial Instrument $\mathcal{E}$ Navigation System Technology Laboratory, \\ Beijing 100191, China
}

Correspondence should be addressed to Xiaolin Ning, ningxiaolin@buaa.edu.cn

Received 15 July 2011; Revised 30 September 2011; Accepted 5 October 2011

Academic Editor: Silvia Maria Giuliatti Winter

Copyright (C) 2012 Xiaolin Ning et al. This is an open access article distributed under the Creative Commons Attribution License, which permits unrestricted use, distribution, and reproduction in any medium, provided the original work is properly cited.

Satellite autonomous orbit determination (OD) is a complex process using filtering method to integrate observation and orbit dynamic equations effectively and estimate the position and velocity of a satellite. Therefore, the filtering method plays an important role in autonomous orbit determination accuracy and time consumption. Extended Kalman filter (EKF), unscented Kalman filter (UKF), and unscented particle filter (UPF) are three widely used filtering methods in satellite autonomous $\mathrm{OD}$, owing to the nonlinearity of satellite orbit dynamic model. The performance of the system based on these three methods is analyzed under different conditions. Simulations show that, under the same condition, the UPF provides the highest OD accuracy but requires the highest computation burden. Conclusions drawn by this study are useful in the design and analysis of autonomous orbit determination system of satellites.

\section{Introduction}

Orbit determination (OD) of satellite plays a significant role in satellite missions, aiming at estimating the ephemeris of a satellite at a chosen epoch accurately. To date, the conventional OD system is dominated by measurements based on (1) ground tracking approaches [1] such as range, range rate, and angle, and (2) Global Position System measurement $[2,3]$. The orbit determination technologies have shown fair performance on various space missions. However, its high cost, lack of robustness to loss of contact, space segment degradation, and 


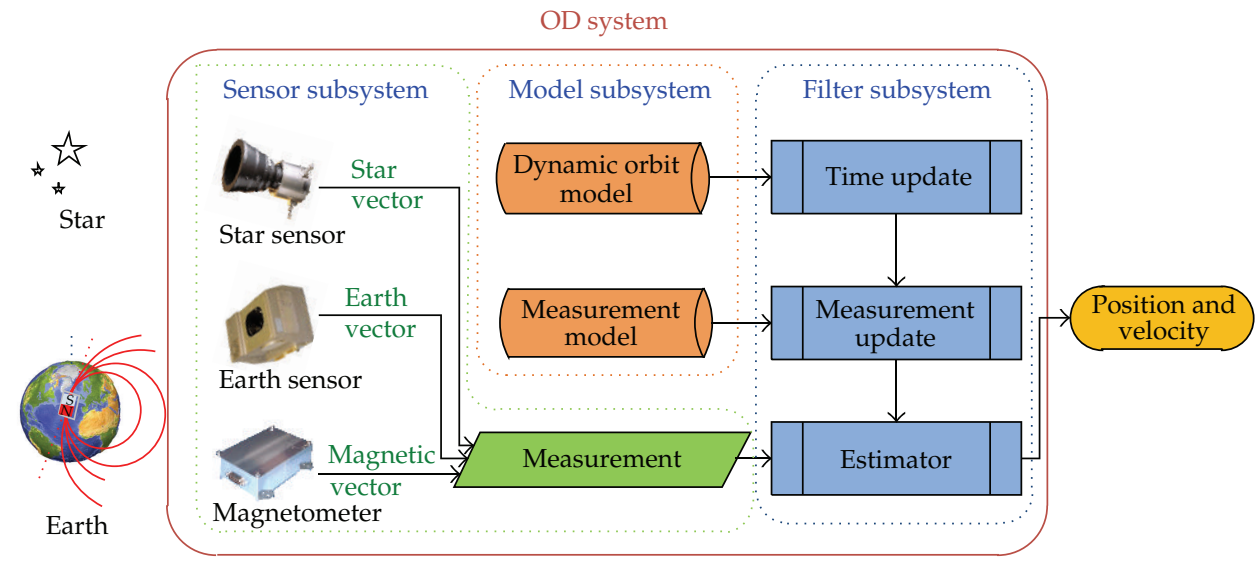

Figure 1: The process of orbit determination.

other factors promote the application of autonomous OD system, which is less costly and less vulnerable in hostile environment [4].

In general, orbit determination is the process of estimating the satellite's state variables (position and velocity) by comparing (in statistical sense) the difference between the measurement data and the estimated data. Orbit determination system, as shown in Figure 1, usually includes sensor subsystem, model subsystem, and filter subsystem. Sensor subsystem contains sensing instruments, such as star sensor, earth sensor, and magnetometer, in order to measure and process the original measurements which are functions of state variables. Model system generates estimated data including state model and measurement model. In the filter subsystem, the optimal algorithms (filtering methods) process both data from sensor subsystem and from model subsystem and then estimate state variables.

Owing to the nonlinear dynamic model of satellite orbit motion, the filtering method applied in OD system should be appropriate for nonlinear system [5, 6]. Extended Kalman filter (EKF), unscented Kalman filter (UKF), and unscented particle filter (UPF) are three main methods used in satellite OD system. The EKF is based on the analytical Taylor series expansion of the nonlinear systems and measurement equations. It works on the principle that the state distribution is approximated by a Gaussian random variable. However, the Taylor series approximations in EKF introduce large errors due to the neglected nonlinearities [7]. The UKF uses the true nonlinear model and a set of sigma sample points produced by the unscented transformation to capture the mean and covariance of state, but the UKF has the limitation that it does not apply to general non-Gaussian distribution $[8,9]$. The particle filter $(\mathrm{PF})$ is a computer-based method for implementing a recursive Bayesian filter by Monte Carlo simulations. The performance of the PF largely depends on the choices of importance sampling density and resampling scheme $[10,11]$. Among many improved PF methods, UPF is a hybrid of the UKF and the particle filter which uses the UKF to get better importance sampling density $[12,13]$. It combines the merits of unscented transformation and particle filtering and avoids their limitations.

A variety of autonomous orbit determination methods have been proposed and explored, including a magnetometer-based OD method [14, 15], a celestial OD method $[16,17]$, a landmark OD method [18, 19], and an X-ray pulsar OD method [20, 21]. The first two methods can be used in low earth orbit (LEO) satellite autonomous orbit determination system. Thus, in this paper, these two OD methods are selected for analysis. 
This paper is divided into five sections. After this introduction, the basic descriptions of three filtering methods in autonomous OD system are given in Section 2. Then, the state model and measurement models in OD model subsystem are described in detail in Section 3. In Section 4, simulations are shown for analyzing and comparing three filtering methods. Finally, conclusions are drawn in Section 5.

\section{Filtering Methods}

The best known algorithms to solve the problem of autonomous satellite orbit determination are the EKF, UKF, and UPF. In this section, we shall present the theories of the three filter algorithms. These algorithms will be incorporated into the filtering framework based on the dynamic state-space model as follows:

$$
\begin{gathered}
\mathbf{x}_{k}=f\left(\mathbf{x}_{k-1}, k-1\right)+\mathbf{w}_{k}, \\
\mathbf{z}_{k}=h\left(\mathbf{x}_{k}, k\right)+\mathbf{v}_{k},
\end{gathered}
$$

where $\mathbf{x}_{k-1}$ denotes the state of the system at time $k-1, \mathbf{z}_{k}$ denotes the observations at step $k$, $\mathbf{w}_{k}$ denotes the process noise, and $\mathbf{v}_{k}$ denotes the measurement noise. The mappings $f$ and $h$ represent the process and measurement models. $\mathbf{E}\left(\mathbf{w}_{k} \mathbf{w}_{j}^{T}\right)=\mathbf{Q}_{k}, \mathbf{E}\left(\mathbf{v}_{k} \mathbf{v}_{j}^{T}\right)=\mathbf{R}_{k}$, for all $k, j$, and $\mathbf{Q}_{k}$ is the process noise covariance at step $k, \mathbf{R}_{k}$ is the measurement noise covariance at step $k$.

\subsection{Extended Kalman Filter}

A Kalman filter that linearizes about the current mean and covariance is referred to as an extended Kalman filter or EKF. The EKF is the minimum mean-square-error estimator based on the Taylor series expansion of the nonlinear functions. For example,

$$
f\left(\mathbf{x}_{k}\right)=f\left(\widehat{\mathbf{x}}_{k \mid k-1}\right)+\left.\frac{\partial f\left(\mathbf{x}_{k}\right)}{\partial \mathbf{x}_{k}}\right|_{\mathbf{x}_{k}=\widehat{\mathbf{x}}_{k \mid k-1}}\left(\mathbf{x}_{k}-\widehat{\mathbf{x}}_{k \mid k-1}\right)+\cdots .
$$

Using only the linear expansion terms, it is easy to derive the update equations for the mean and covariance of the Gaussian approximation to the distribution of the states [12].

The equations for the extended Kalman filter fall into two groups: time update equations and measurement update equations. The specific equations for the time and measurement updates are presented below as shown in (2.3) (2.8) [22].

(1) Time Update

Predicted state estimate:

$$
\widehat{\mathbf{x}}_{k \mid k-1}=f\left(\widehat{\mathbf{x}}_{k-1}, k-1\right) .
$$

Predicted estimate covariance:

$$
\mathbf{P}_{k}^{-}=\mathbf{\Phi}_{k} \mathbf{P}_{k-1} \mathbf{\Phi}_{k}^{T}+\mathbf{Q}_{k-1}
$$


The time update equations project the state, $\mathbf{x}_{k}$, and covariance, $\mathbf{P}_{k}$, estimates from the previous time step $k-1$ to the current time step $k, \mathbf{\Phi}_{k}$ is the state transition matrix at step $k$, which is defined to be the following Jacobians:

$$
\mathbf{\Phi}_{k}=\left.\frac{\partial f}{\partial \mathbf{x}_{k}}\right|_{\mathbf{x}_{k}=\widehat{\mathbf{x}}_{k \mid k-1}} .
$$

(2) Measurement Update

Near-Optimal Kalman gain:

$$
\mathbf{K}_{k}=\mathbf{P}_{k}^{-1} \mathbf{H}_{k}^{T}\left(\mathbf{H}_{k} \mathbf{P}_{k}^{-1} \mathbf{H}_{k}^{T}+\mathbf{R}_{k}^{-1}\right)^{-1} .
$$

Updated state estimate:

$$
\widehat{\mathbf{x}}_{k}=\widehat{\mathbf{x}}_{k \mid k-1}+\mathbf{K}_{k}\left(\mathbf{z}_{k}-h\left(\widehat{\mathbf{x}}_{k \mid k-1}, k\right)\right) .
$$

Updated estimate covariance:

$$
\mathbf{P}_{k}=\left(\mathbf{I}-\mathbf{K}_{k} \mathbf{H}_{k}\right) \mathbf{P}_{k}^{-}
$$

where $\mathbf{K}_{k}$ is known as the Kalman gain. The measurement update equations correct the state and covariance estimates with the measurement $\mathbf{z}_{k}$. $\mathbf{H}_{k}$ is the observation matrix at step $k$, which is defined to be the following Jacobians:

$$
\mathbf{H}_{k}=\left.\frac{\partial h}{\partial \mathbf{x}_{k}}\right|_{\mathbf{x}_{k}=\widehat{\mathbf{x}}_{k \mid k-1}} .
$$

The major drawback of EKF is that it only uses the first order terms in the Taylor series expansion. Sometimes it may introduce large estimation errors in a nonlinear system and lead to poor representations of the nonlinear functions and probability distributions of interest. As a result, this filter can diverge [23].

\subsection{Unscented Kalman Filter}

The unscented Kalman filter (UKF) $[8,24]$ uses the unscented transformation to capture the mean and covariance estimates with a minimal set of sample points. The UKF process is identical to the standard EKF process with the prediction-estimation recursive loop. The exception is that the UKF uses the sigma points and the nonlinear equations to compute the predicted states and measurements and the associated covariance matrices. If the dimension of state is $n \times 1$, the $2 n+1$ sigma point and their weight are computed by [9]

$$
\begin{gathered}
\mathbf{X}_{0, k}=\widehat{\mathbf{x}}_{k}, \quad W_{0}=\frac{\tau}{(n+\tau)}, \\
\mathbf{X}_{i, k}=\widehat{\mathbf{x}}_{k}+\sqrt{n+\tau}(\sqrt{\mathbf{P}(k \mid k)})_{i}^{\prime} \quad W_{i}=\frac{1}{[2(n+\tau)]}, \quad i=1,2, \ldots, n, \\
\mathbf{X}_{i+n, k}=\widehat{\mathbf{x}}_{k}-\sqrt{n+\tau}(\sqrt{\mathbf{P}(k \mid k)})_{i}, \quad W_{i+n}=\frac{1}{[2(n+\tau)]},
\end{gathered}
$$


where $\tau \in \mathbf{R},(\sqrt{\mathbf{P}(k \mid k)})_{i}$ is the $i$ th column of the matrix square root. The UKF process can be described as follows.

(1) Time $=0$, initialize the UKF with $\widehat{\mathbf{x}}_{0}$ and $\mathbf{P}_{0}$ as follows:

$$
\begin{gathered}
\widehat{\mathbf{x}}_{0}=E\left[\mathbf{x}_{0}\right], \\
\mathbf{P}_{0}=E\left[\left(\mathbf{x}_{0}-\widehat{\mathbf{x}}_{0}\right)\left(\mathbf{x}_{0}-\widehat{\mathbf{x}}_{0}\right)^{T}\right] .
\end{gathered}
$$

(2) Time $=k$, define $2 n+1$ sigma points from

$$
\mathbf{X}_{k-1}=\left[\begin{array}{lll}
\mathbf{X}_{0, k} & \mathbf{X}_{i, k} & \mathbf{X}_{i+n, k}
\end{array}\right], \quad i=1,2, \ldots, n .
$$

The equations for the UKF fall into two groups the same as EKF: time update equations and measurement update equations. The specific equations for the time and measurement updates are presented below.

(1) Time Update

$$
\begin{gathered}
\mathbf{X}_{k \mid k-1}=f\left(\mathbf{X}_{k-1}, k-1\right), \\
\widehat{\mathbf{x}}_{k}^{-}=\sum_{i=0}^{2 n} W_{i} \mathbf{X}_{i, k \mid k-1}, \\
\mathbf{P}_{k}^{-}=\sum_{i=0}^{2 n} W_{i}\left[\mathbf{X}_{i, k \mid k-1}-\widehat{\mathbf{x}}_{k}^{-}\right] \cdot\left[\mathbf{X}_{i, k \mid k-1}-\widehat{\mathbf{x}}_{k}^{-}\right]^{T}+\mathbf{Q}_{k}, \\
\mathbf{Z}_{k \mid k-1}=h\left(\mathbf{X}_{k \mid k-1}, k\right), \\
\widehat{\mathbf{z}}_{k}^{-}=\sum_{i=0}^{2 n} W_{i} \mathbf{Z}_{i, k \mid k-1} .
\end{gathered}
$$

(2) Measurement Update

$$
\begin{gathered}
\mathbf{P}_{\widehat{z}_{k} \widehat{z}_{k}}=\sum_{i=0}^{2 n} W_{i}\left[\mathbf{Z}_{i, k \mid k-1}-\widehat{\mathbf{z}}_{k}^{-}\right]\left[\mathbf{Z}_{i, k \mid k-1}-\widehat{\mathbf{z}}_{k}^{-}\right]^{T}+\mathbf{R}_{k}, \\
\mathbf{P}_{\widehat{x}_{k} \hat{z}_{k}}=\sum_{i=0}^{2 n} W_{i}\left[\mathbf{X}_{i, k \mid k-1}-\widehat{\mathbf{x}}_{k}^{-}\right]\left[\mathbf{Z}_{i, k \mid k-1}-\widehat{\mathbf{z}}_{k}^{-}\right]^{T}, \\
\mathbf{K}_{k}=\mathbf{P}_{\widehat{x}_{k} \widehat{z}_{k}} \mathbf{P}_{\widehat{z}_{k} \widehat{z}_{k^{\prime}}}^{-1} \\
\widehat{\mathbf{x}}_{k}=\widehat{\mathbf{x}}_{k}^{-}+\mathbf{K}_{k}\left(\mathbf{z}_{k}-\widehat{\mathbf{z}}_{k}^{-}\right), \\
\mathbf{P}_{k}=\mathbf{P}_{k}^{-}-\mathbf{K}_{k} \mathbf{P}_{\widehat{z}_{k} \widehat{z}_{k}} \mathbf{K}_{k}^{T} .
\end{gathered}
$$




\subsection{Unscented Particle Filter}

The unscented particle filter (UPF) is a hybrid of the UKF and the particle filter which uses the UKF to get better importance sampling density. A pseudo-code description of UPF is as follows [11-13].

(1) Initialization: Time $=0$.

Generate $N$ samples $\mathbf{x}_{0^{\prime}}^{i}(i=1,2, \ldots, N)$ from the prior $p\left(\mathbf{x}_{0}\right)$, and set the importance weight $w_{0}^{i}$ of each sample $1 / N$ :

$$
\widehat{\mathbf{x}}_{0}^{i}=E\left[\mathbf{x}_{0}^{i}\right], \quad \mathbf{P}_{0}^{i}=E\left[\left(\mathbf{x}_{0}^{i}-\widehat{\mathbf{x}}_{0}^{i}\right)\left(\mathbf{x}_{0}^{i}-\widehat{\mathbf{x}}_{0}^{i}\right)^{T}\right], \quad w_{0}^{i}=\frac{1}{N}
$$

(2) Time $=k$.

(I) (a) Update the particles with the UKF:

(i) calculate sigma points from $\left\{\widehat{\mathbf{x}}_{k-1}^{i}, \mathbf{P}_{k-1}^{i}\right\}$ using (2.12),

(ii) propagate particle into future by (2.13),

(iii) incorporate new observation to update the measurement by (2.14) and obtain $\left\{\widehat{\mathbf{x}}_{k}^{i}, \mathbf{P}_{k}^{i}\right\}$.

(b) Sample a new particle $\widehat{\mathbf{x}}_{k}^{i}$ and make $\widehat{\mathbf{x}}_{k}^{i} \sim q\left(\mathbf{x}_{k}^{i} \mid \mathbf{x}_{k-1}^{i}, \mathbf{z}_{k}\right)=N\left(\widehat{\mathbf{x}}_{k}^{i}, \mathbf{P}_{k}^{i}\right)$.

(II) Compute the importance weight $\bar{w}_{k}^{i}$ and normalize the importance weights $w_{k}^{i}$ :

$$
\begin{gathered}
\bar{w}_{k}^{i}=w_{k-1}^{i} \cdot \frac{p\left(\mathbf{z}_{k} \mid \widehat{\mathbf{x}}_{k}^{i}\right) p\left(\widehat{\mathbf{x}}_{k}^{i} \mid \mathbf{x}_{k-1}^{i}\right)}{q\left(\widehat{\mathbf{x}}_{k}^{i} \mid \mathbf{x}_{k-1}^{i}, \mathbf{z}_{k-1}\right)}, \\
w_{k}^{i}=\frac{\bar{w}_{k}^{i}}{\sum_{i=1}^{N} \bar{w}_{k}^{i}},
\end{gathered}
$$

where $p\left(\mathbf{z}_{k} \mid \widehat{\mathbf{x}}_{k}^{i}\right)$ is likelihood probability distribution, which is given by measurement model $\mathbf{z}_{k}=h\left(\mathbf{x}_{k}, k\right)+\mathbf{v}_{k}, p\left(\widehat{\mathbf{x}}_{k}^{i} \mid \mathbf{x}_{k-1}^{i}\right)$ is the forward transition probability distribution, which is given by process model $\mathbf{x}_{k}=f\left(\mathbf{x}_{k-1}, k-1\right)+\mathbf{w}_{k}, q\left(\widehat{\mathbf{x}}_{k}^{i} \mid \mathbf{x}_{k-1}^{i}, \mathbf{z}_{k-1}\right)$ is the proposal distribution [12].

(III) Resampling step:

The basic idea of resampling is to eliminate particles with small weights and to concentrate on particles with large weights. Multiply/suppress particles $\left\{\widehat{\mathbf{x}}_{k^{\prime}}^{i} \mathbf{P}_{k}^{i}\right\}$ with high/low importance weights $w_{k^{\prime}}^{i}$, respectively, to obtain $N$ random particles $\left\{\tilde{\mathbf{x}}_{k^{\prime}}^{i} \tilde{\mathbf{P}}_{k}^{i}\right\}$.

(IV) Output step:

The overall state estimation and covariance are

$$
\begin{gathered}
\widehat{\mathbf{x}}_{k}=\sum_{i=1}^{N} w_{k}^{i} \widetilde{\mathbf{x}}_{k \prime}^{i} \\
\mathbf{P}_{k}=\sum_{i=1}^{N} w_{k}^{i} \widetilde{\mathbf{P}}_{k}^{i}=\sum_{i=1}^{N} w_{k}^{i}\left(\widetilde{\mathbf{x}}_{k}^{i}-\widehat{\mathbf{x}}_{k}\right)\left(\widetilde{\mathbf{x}}_{k}^{i}-\widehat{\mathbf{x}}_{k}\right)^{T}
\end{gathered}
$$




\section{System Models}

\subsection{State Model}

The state model (dynamical model) of the celestial OD system for a near-Earth satellite based on the orbital dynamics in the Earth-Centered Inertial (ECI) frame (J2000.0) is

$$
\begin{gathered}
\frac{d x}{d t}=v_{x}, \quad \frac{d y}{d t}=v_{y}, \quad \frac{d z}{d t}=v_{z} \\
\frac{d v_{x}}{d t}=-\frac{\mu x}{r^{3}} \cdot\left[1-J_{2}\left(\frac{R_{e}}{r}\right)^{2}\left(\frac{7.5 z^{2}}{r^{2}}-1.5\right)\right]+\Delta F_{x} \\
\frac{d v_{y}}{d t}=-\frac{\mu y}{r^{3}} \cdot\left[1-J_{2}\left(\frac{R_{e}}{r}\right)^{2}\left(\frac{7.5 z^{2}}{r^{2}}-1.5\right)\right]+\Delta F_{y}, \\
\frac{d v_{z}}{d t}=-\frac{\mu z}{r^{3}} \cdot\left[1-J_{2}\left(\frac{R_{e}}{r}\right)^{2}\left(\frac{7.5 z^{2}}{r^{2}}-4.5\right)\right]+\Delta F_{z} \\
r=\sqrt{x^{2}+y^{2}+z^{2}} .
\end{gathered}
$$

Equation (3.1) can be written in a general state equation as

$$
\dot{\mathbf{X}}(t)=f(\mathbf{X}(t), t)+\mathbf{w}(t),
$$

where $\mathbf{X}=\left[\begin{array}{llllll}x & y & z & v_{x} & v_{y} & v_{z}\end{array}\right]^{T}$ is the state vector. $x, y, z, v_{x}, v_{y}, v_{z}$ are satellite positions and velocities of the three axes, respectively, $\mu$ is the gravitational constant of earth, $J_{2}$ is the second zonal coefficient and has the value 0.0010826269 [25], and $R_{e}$ is the earth's radius. $\Delta F_{x}, \Delta F_{y}, \Delta F_{z}$ are the perturbations including high order nonspherical earth perturbations, third-body perturbations, atmospheric drag perturbations, solar radiation perturbations, and other perturbations, which are considered as process noises $\mathbf{w}(t)$.

\subsection{Celestial Orbit Determination and Its Measurement}

The celestial OD method is based on the fact that the position of a celestial body in the inertial frame at a certain time is known and that its position measured in the spacecraft body frame is a function of the satellite's position. To earth satellite, stars are distributed all over the sky, and the positions of Earth are fixed at a certain time. The geometric relationship among stars, the Earth, and satellite enables us to determine the position of the satellite [26].

Satellite celestial OD methods can be broadly separated into two major approaches: directly sensing horizon method and indirectly sensing horizon method. In this paper, the directly sensing horizon method is used.

The angle between a star and the earth, $\alpha$, as shown in Figure 2, is a kind of directly sensing horizon measurement of satellite celestial OD system, which is measured by star sensor and earth sensor. The measurement model using the star-earth angle is given by [27]

$$
\alpha=\arccos \left(\frac{-\mathbf{s} \cdot \mathbf{r}}{r}\right)+v_{\alpha}
$$




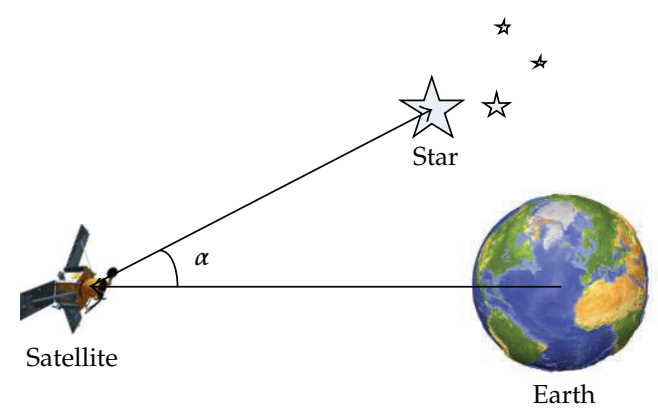

Figure 2: The measurement of celestial OD system.

where $\mathbf{r}$ is the position vector of the satellite, which is the same as that in (3.2), $\mathbf{s}$ is the position vector of the star in the earth-centered inertial frame, $v_{\alpha}$ is the measurement noise.

Assuming a measurement $Z_{1}=[\alpha]$ and measurement noise $V_{1}=\left[v_{\alpha}\right],(3.2)$ can be written as a general measurement equation

$$
\mathbf{Z}_{1}(t)=h_{1}[\mathbf{X}(t), t]+\mathbf{V}_{1}(t)
$$

\subsection{Geomagnetic Orbit Determination and Its Measurement}

Geomagnetic OD system relies on measurements from a three-axis magnetometer to determine satellite position and orbit. It uses a model of Earth's magnetic field and a model of orbital dynamics to predict the time-varying magnitude of Earth's magnetic field vector at the space. OD system compares the time history of the predicted magnitude and the measured magnitude time history in filter sense to obtain the optimal estimated state (position and velocity) [14].

\subsubsection{Magnetic Model}

Two main models used for describing Earth's magnetic vector in the geodetic reference frame are World Magnetic Model (WMM) and International Geomagnetic Reference Field (IGRF) [28]. The WMM 2005 is selected in this paper for geomagnetic orbit determination [29].

According to the WMM model 2005, the vector field $\mathbf{B}$ can be written as the gradient of a potential function

$$
\mathbf{B}(r, \lambda, \theta, t)=-\nabla V(r, \lambda, \theta, t),
$$

where $(r, \lambda, \theta)$ represent the radius, the longitude, and the colatitude in a spherical, geocentric reference frame, respectively.

This potential $V$ can be expanded in terms of spherical harmonics:

$$
V(r, \lambda, \theta, t)=\sum_{n=1}^{N} \sum_{m=0}^{n} V_{n}^{m}=a \sum_{n=1}^{N}\left(\frac{a}{r}\right)^{n+1} \sum_{m=0}^{n}\left[g_{n}^{m}(t) \cos (m \lambda)+h_{n}^{m}(t) \sin (m \lambda)\right] \breve{P}_{n}^{m}(\cos \theta),
$$



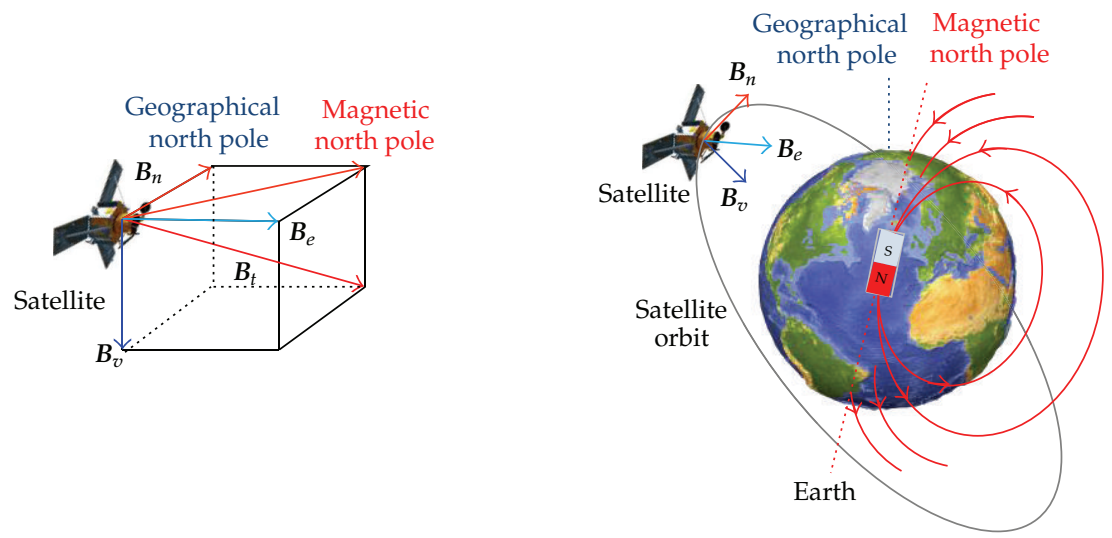

Figure 3: The measurement of geomagnetic OD system.

where $N=12$ is the degree of the expansion of the WMM, $a$ is the standard Earth's magnetic reference radius, $g_{n}^{m}(t)$ and $h_{n}^{m}(t)$ are the time-dependent Gauss coefficients of degree $n$ and order $m$, and $\breve{P}_{n}^{m}(\cos \theta)$ are the Schmidt normalized associated Legendre polynomials.

\subsubsection{Magnetic Measurement Model}

Based on the relationship between magnetic vector, which is obtained by the magnetometer, and the earth magnetic model, the measurement model can be written as

$$
\mathbf{B}_{s}=\mathbf{A}_{\mathrm{sb}} \mathbf{A}_{\mathrm{bi}} \mathbf{A}_{\mathrm{it}} \mathbf{B}_{t}+\mathbf{v}_{B}
$$

where $\mathbf{B}_{s}$ is the magnetic vector of local position in sensor coordinates, which can be obtained from vector magnetometer system consisting of three mutually orthogonal, singleaxis magnetometers. $\mathbf{B}_{t}=\left[\begin{array}{lll}B_{n} & B_{e} & B_{v}\end{array}\right]^{T}$ is the magnetic vector of local position in geocentric coordinates, and it can be obtained from WMM according to local longitude, latitude, and height, as shown in Figure 3; $\mathbf{A}_{\mathrm{sb}}, \mathbf{A}_{\mathrm{bi}}$, and $\mathbf{A}_{\mathrm{it}}$ are the transformation matrices from satellite body coordinates to sensor coordinates, from earth inertial coordinates to satellite body coordinates, and from earth inertial coordinates to geocentric coordinates, respectively. $\mathbf{v}_{B}$ is the measurement noise.

Assuming a measurement $\mathbf{Z}_{2}=\mathbf{B}_{s}$ and measurement noise $\mathbf{V}_{2}=\mathbf{v}_{B}$, (3.7) can be written as a general measurement equation as

$$
\mathbf{Z}_{2}(t)=h_{2}[\mathbf{X}(t), t]+\mathbf{V}_{2}(t)
$$

\section{Analysis and Comparison}

\subsection{Simulation Condition}

The trajectory used in the following simulation is a LEO satellite whose orbital parameters are semimajor axis $a=7136.635444 \mathrm{~km}$, eccentricity $e=1.809 \times 10^{-3}$, inclination $i=65^{\circ}$, right ascension of the ascending node $\Omega=30^{\circ}$, and the argument of perigee $\omega=30^{\circ}$. The orbit 


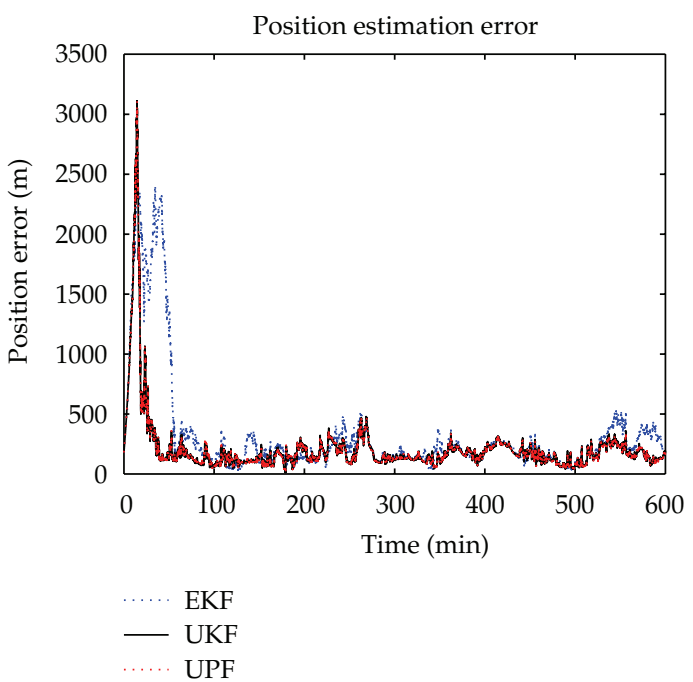

(a)

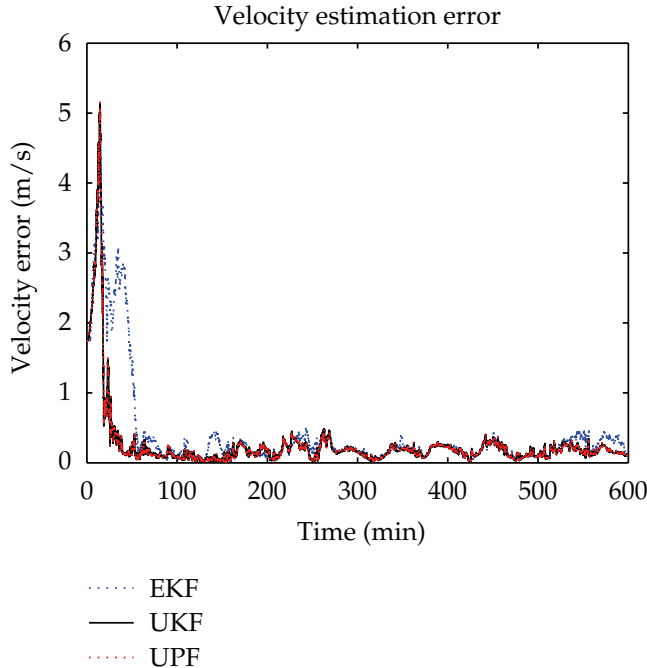

(b)

Figure 4: Three filtering methods results of celestial OD system $(T=3 \mathrm{~s})$.

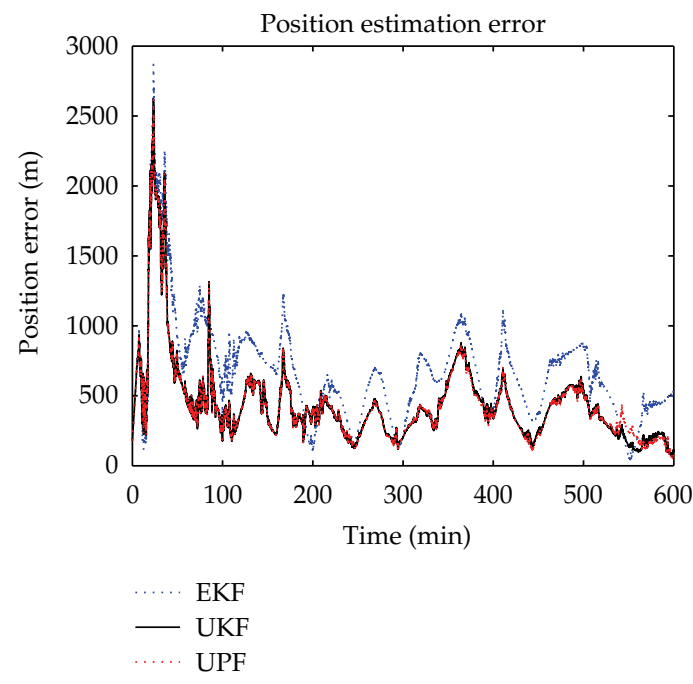

(a)

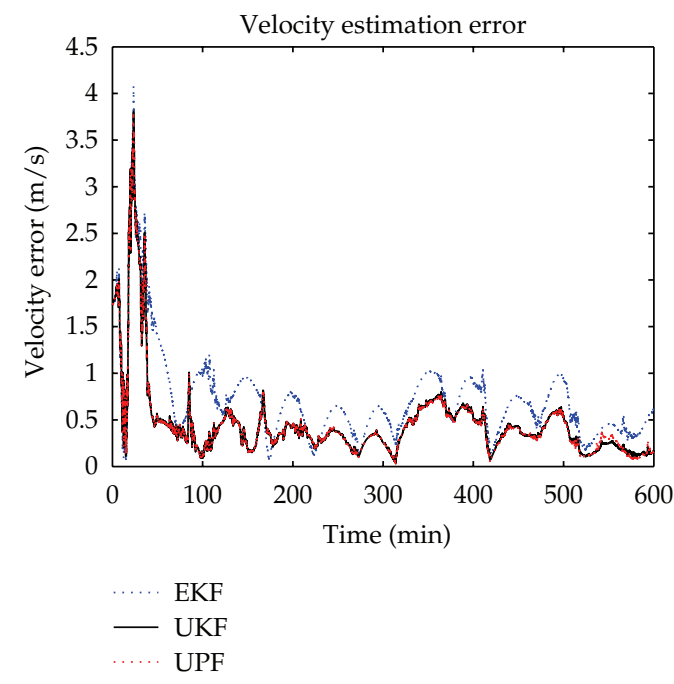

(b)

Figure 5: Three filtering methods results of geomagnetic OD system $(T=3 \mathrm{~s})$.

and attitude data of the satellite are produced by the Satellite Tool Kit (STK) software [30]. The accuracy of star sensor and earth sensor is selected $3^{\prime \prime}$ and $0.02^{\circ}$, respectively. The stellar database used in simulation is the Tycho stellar catalog [31]. The magnetometer measurement and geomagnetic model accuracy is considered as $100 \mathrm{nT}$ [32].

\subsection{Performances under Different Sampling Intervals}

Figures 4 and 5 show the performances comparison among the EKF, UKF, and UPF methods of celestial OD system and geomagnetic OD system, respectively. Data is obtained with a $3 \mathrm{~s}$ 
Table 1: Performance of celestial OD system under different sampling intervals.

\begin{tabular}{cccccc}
\hline \multirow{2}{*}{ Sampling interval } & \multicolumn{2}{c}{ RMS (after convergence) } & \multicolumn{2}{c}{ Maximum (after convergence) } \\
& & Position error/m & Velocity error/m/s & Position error/m & Velocity error/m/s \\
\hline \multirow{2}{*}{$T=3 \mathrm{~s}$} & EKF & 203.318211 & 0.196622 & 532.987272 & 0.486705 \\
& UKF & 161.312723 & 0.162900 & 357.545600 & 0.407503 \\
& UPF & 159.756079 & 0.160780 & 354.555359 & 0.402378 \\
\hline \multirow{2}{*}{$T=15 \mathrm{~s}$} & EKF & 271.640953 & 0.287641 & 703.000581 & 0.613708 \\
& UKF & 245.939302 & 0.219864 & 593.829098 & 0.563749 \\
& UPF & 245.229683 & 0.219566 & 593.802048 & 0.563396 \\
$T=60 \mathrm{~s}$ & EKF & 934.238939 & 0.976641 & 2457.895170 & 2.348656 \\
& UKF & 736.876288 & 0.699942 & 2322.291896 & 2.302674 \\
& UPF & 735.166932 & 0.698808 & 2314.761249 & 2.294924 \\
\hline
\end{tabular}

Table 2: Performance of geomagnetic OD system under different sampling intervals.

\begin{tabular}{cccccc}
\hline \multirow{2}{*}{ Sampling interval } & \multicolumn{2}{c}{ RMS (after convergence) } & \multicolumn{2}{c}{ Maximum (after convergence) } \\
& & Position error/m & Velocity error/m/s & Position error/m & Velocity error/m/s \\
\hline \multirow{2}{*}{$T=3 \mathrm{~s}$} & EKF & 591.253628 & 0.601946 & 1129.365510 & 1.061735 \\
& UKF & 376.894372 & 0.371566 & 877.909403 & 0.799659 \\
& UPF & 376.516863 & 0.366538 & 861.513975 & 0.783449 \\
\hline \multirow{2}{*}{$T=15 \mathrm{~s}$} & EKF & 1481.673752 & 1.358867 & 2851.660177 & 2.607626 \\
& UKF & 705.765450 & 0.648228 & 1325.501267 & 1.276198 \\
& UPF & 705.161263 & 0.647876 & 1323.976107 & 1.274585 \\
$T=60 \mathrm{~s}$ & EKF & 4343.783162 & 4.308921 & 14643.275741 & 11.712547 \\
& UKF & 3904.890544 & 3.633798 & 10403.528541 & 10.328902 \\
& UPF & 3904.747892 & 3.633460 & 10401.279139 & 10.328554 \\
\hline
\end{tabular}

sampling interval during the $600 \mathrm{~min}$ period ( 6 orbits). Tables 1 and 2 present the details of the simulation results of celestial OD system and geomagnetic OD system under different sampling intervals, respectively.

The simulations in Figures 4 and 5 suggest that the EKF-based OD system performance is the worst. In contrast, UPF-based OD system provides the highest OD accuracy. As the details in Tables 1 and 2, regardless the celestial OD and geomagnetic OD system, the different sampling intervals can strongly affect the OD accuracy. OD performance is degraded remarkably with increasing sample interval. However, under the same sampling interval, the EKF method is the most sensitive to the sampling interval, for the nonlinear error increases rapidly with the longer sampling interval. In contrast, the UKF and UPF perform distinctly better.

\subsection{Performance under Different Noise Distributions}

This subsection reports how different noise distributions affect the OD performances using three filters. We selected three common noise distributions in navigation, and they are normal distribution, student's $t$ distribution, and uniform distribution [33]. 


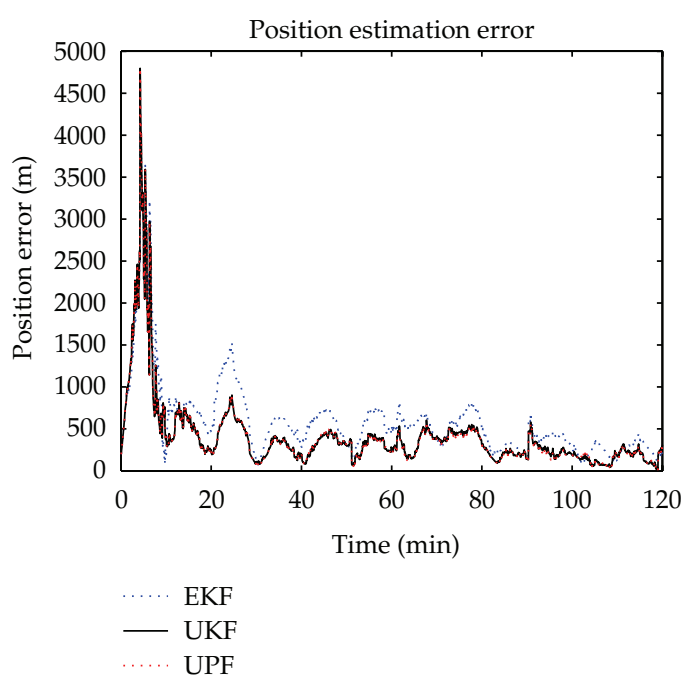

(a)

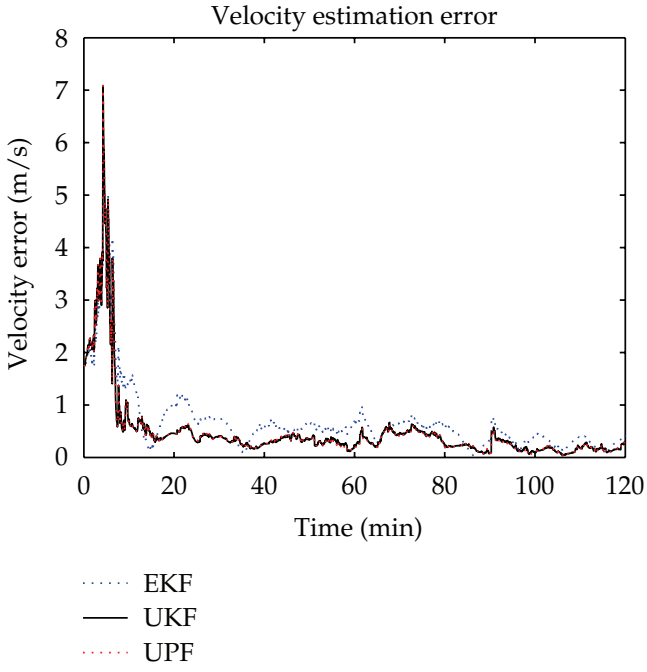

(b)

Figure 6: Celestial OD results of three filtering methods under Student's $t$ noise distributions.

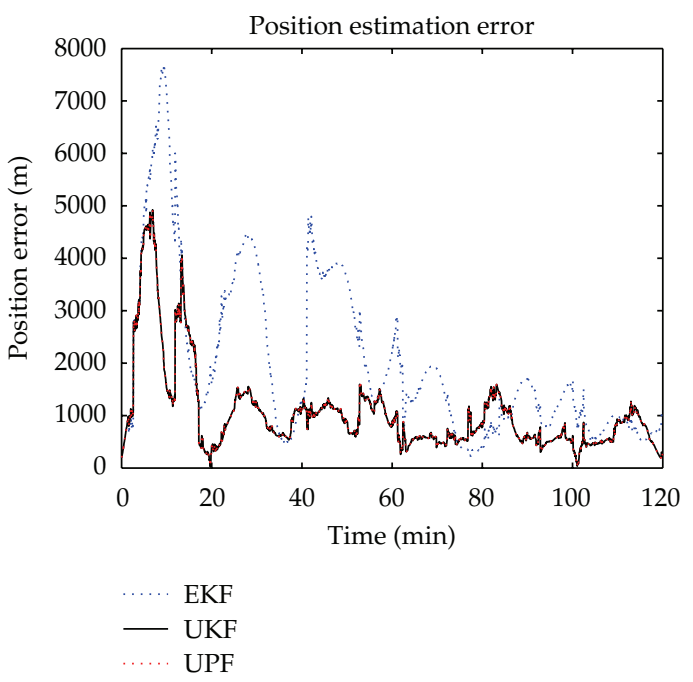

(a)

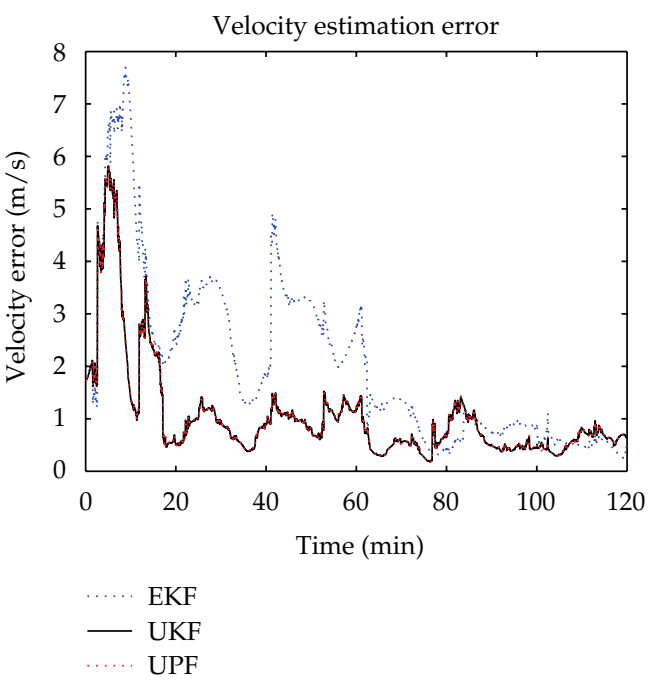

(b)

Figure 7: Geomagnetic OD results of three filtering methods under Student's $t$ noise distributions.

Figures 6 and 7 show the OD results of celestial OD system and geomagnetic OD system using three filters under student's $t$ noise distributions, respectively. All performance curves were obtained with $15 \mathrm{~s}$ sampling interval during the $600 \mathrm{~min}$ period (6 orbits). Tables 3 and 4 present the details of the simulation results of celestial OD system and geomagnetic OD system under three different noise distributions, respectively.

As the results in Figures 6 and 7 showed, the UPF-based geomagnetic OD system provides the highest OD accuracy. As the details in Tables 3 and 4 demonstrated, OD performance under different noise distribution is similar. In general, the EKF performance 
Table 3: Performance of celestial OD system under different noise distributions.

\begin{tabular}{|c|c|c|c|c|c|}
\hline \multirow{2}{*}{\multicolumn{2}{|c|}{ Noise distribution }} & \multicolumn{2}{|c|}{ RMS (after convergence) } & \multicolumn{2}{|c|}{ Maximum (after convergence) } \\
\hline & & Position error $/ \mathrm{m}$ & Velocity error $/ \mathrm{m} / \mathrm{s}$ & Position error $/ \mathrm{m}$ & Velocity error $/ \mathrm{m} / \mathrm{s}$ \\
\hline \multirow{3}{*}{$\begin{array}{l}\text { Normal } \\
\text { distribution }\end{array}$} & EKF & 271.640953 & 0.287641 & 703.000581 & 0.613708 \\
\hline & UKF & 245.939302 & 0.219864 & 593.829098 & 0.563749 \\
\hline & UPF & 245.229683 & 0.219566 & 593.802048 & 0.563396 \\
\hline \multirow{3}{*}{$\begin{array}{l}\text { Student's } t \\
\text { distribution }\end{array}$} & EKF & 387.618044 & 0.411669 & 827.358300 & 0.972190 \\
\hline & UKF & 257.104360 & 0.261611 & 604.325265 & 0.664447 \\
\hline & UPF & 246.294197 & 0.250511 & 587.154229 & 0.639735 \\
\hline \multirow{3}{*}{$\begin{array}{l}\text { Uniform } \\
\text { distribution }\end{array}$} & EKF & 385.803609 & 0.398842 & 994.714264 & 0.902353 \\
\hline & UKF & 350.079516 & 0.339869 & 888.790677 & 0.788940 \\
\hline & UPF & 349.537325 & 0.338473 & 880.417206 & 0.782641 \\
\hline
\end{tabular}

Table 4: Performance of geomagnetic OD system under different noise distributions.

\begin{tabular}{|c|c|c|c|c|c|}
\hline \multirow{2}{*}{\multicolumn{2}{|c|}{ Noise distribution }} & \multicolumn{2}{|c|}{ RMS (after convergence) } & \multicolumn{2}{|c|}{ Maximum (after convergence) } \\
\hline & & \multirow{2}{*}{$\begin{array}{c}\text { Position error /m } \\
1481.673752\end{array}$} & \multirow{2}{*}{$\frac{\text { Velocity error } / \mathrm{m} / \mathrm{s}}{1.358867}$} & \multirow{2}{*}{$\begin{array}{c}\text { Position error } / \mathrm{m} \\
2851.660177\end{array}$} & \multirow{2}{*}{$\frac{\text { Velocity error } / \mathrm{m} / \mathrm{s}}{2.607626}$} \\
\hline & EKF & & & & \\
\hline $\begin{array}{l}\text { Normal } \\
\text { distribution }\end{array}$ & UKF & 705.765450 & 0.648228 & 1325.501267 & 1.276198 \\
\hline & UPF & 705.161263 & 0.647876 & 1323.976107 & 1.274585 \\
\hline \multirow{3}{*}{$\begin{array}{l}\text { Student's } t \\
\text { distribution }\end{array}$} & EKF & 1059.874849 & 0.828895 & 2900.800178 & 3.184135 \\
\hline & UKF & 670.321661 & 0.604835 & 1594.060692 & 1.436411 \\
\hline & UPF & 669.959004 & 0.604513 & 1593.085809 & 1.436468 \\
\hline \multirow{3}{*}{$\begin{array}{l}\text { Uniform } \\
\text { distribution }\end{array}$} & EKF & 1246.529130 & 1.207728 & 4029.187774 & 3.253167 \\
\hline & UKF & 776.622486 & 0.767562 & 1985.870577 & 1.781823 \\
\hline & UPF & 776.526144 & 0.767452 & 1985.704699 & 1.781594 \\
\hline
\end{tabular}

is the worst and the UPF performance is the best, no matter what measurement errors are chosen.

\subsection{Computation Cost of Three Methods}

Besides the accuracy, the computation cost is another essential requirement to evaluate the performance of filtering methods. Table 5 gives the computation cost of the three methods for the celestial orbit determination system and the geomagnetic orbit determination system, respectively. As in the theoretical value of computation cost, where $\Phi$ is the process Jacobian, $n$ is the order of the $\Phi$, and in the simulation $n$ equals 6 . The simulation results presented here were run on a $2.66 \mathrm{GHz}$ Inter Core2 Duo CPU with 32-bit Windows 7 system. The simulation time of celestial OD system in Table 5 demonstrates that the UPF demands the highest computation time, which is almost twenty times (= sample number) higher than UKF, and EKF requires almost a quarter of the computation time of UKF. However, the simulation time of geomagnetic OD system is not the same amount as celestial OD system, and the EKFbased geomagnetic OD system takes significantly longer time, since the time for computing measurement Jacobians takes a lot of computer resource. 
Table 5: Comparison of computation cost.

\begin{tabular}{|c|c|c|c|c|}
\hline \multirow[b]{2}{*}{$\begin{array}{l}\text { Filter } \\
\text { method }\end{array}$} & \multicolumn{2}{|c|}{ Theoretical value of computation cost } & \multicolumn{2}{|c|}{ Simulation value of computation cos } \\
\hline & $\boldsymbol{\Phi}$ is full matrix & $\boldsymbol{\Phi}$ is diagonal matrix & $\begin{array}{l}\text { Computation } \\
\text { time per orbit } \\
\text { of celestial } \\
\text { OD system(s) }\end{array}$ & $\begin{array}{l}\text { Computation } \\
\text { time per orbit } \\
\text { of geomagnetic } \\
\text { OD system (s) }\end{array}$ \\
\hline EKF & $C_{\mathrm{EKF}}=3 n^{3}+3 n^{2}+4 n$ & $C_{\mathrm{EKF}}^{\prime}=6 n^{2}+4 n$ & 0.3 & 9.5 \\
\hline UKF & $C_{\mathrm{UKF}}=2 n^{3}+12 n^{2}+14 n+5$ & $C_{\mathrm{UKF}}^{\prime}=2 n^{3}+12 n^{2}+14 n+5$ & 1.6 & 11.7 \\
\hline $\begin{array}{l}\text { UPF } \\
\text { (sample } \\
\text { num = 20) }\end{array}$ & $C_{\mathrm{UPF}}=$ sample num $\cdot C_{\mathrm{UKF}}$ & $C_{\mathrm{UPF}}^{\prime}=$ sample num $\cdot C_{\mathrm{UKF}}^{\prime}$ & 39.5 & 313.9 \\
\hline
\end{tabular}

\section{Conclusion}

The problem of choosing a suitable filtering method for the orbit determination application has been studied here. Three filtering methods for the autonomous orbit determination using either celestial or geomagnetic measurements have been studied and their performances have been compared for the estimation problem.

The algorithms are tested with STK satellite orbit data, and the simulation results demonstrate that UPF yields the best OD accuracy and the EKF yields the worst under the same condition. The main reason is that the state equations and measurement equations for autonomous orbit determination system are significantly nonlinear as well as the nonGaussian errors.

In addition, the paper analyzed the computation cost of the three filtering methods, and UPF-based OD system can provide the highest OD accuracy, though it requires the largest computation time. However, the UPF can finally meet the real-time requirements, as with the development of computer technology.

\section{Acknowledgments}

The work described in this paper was supported by the National Natural Science Foundation of China (60874095) and Hi-Tech Research and Development Program of China. The authors would like to thank all members of Science and Technology on Inertial Laboratory and Fundamental Science on Novel Inertial Instrument \& Navigation System Technology Laboratory, for their useful comments regarding this work effort.

\section{References}

[1] D.-J. Lee and K.T. Alfriend, "Precise real-time satellite orbit estimation using the unscented kalman filter," AAS/AIAA Space Flight Mechanics Symposium AAS 03-230, pp. 853-1872, Advances in the Astronautical Sciences, Ponce, Puerto Rico, 2003.

[2] P. C. P. M. Pardal, H. K. Kuga, and R. V. de Moraes, "Recursive least squares algorithms applied to satellite orbit determination, using GPS signals," in Proceedings of the 8th International Conference on Signal Processing, Robotics and Automation, pp. 167-172, World Scientific and Engineering Academy and Society, Cambridge, UK, 2009.

[3] O. Montenbruck and P. Ramos-Bosch, "Precision real-time navigation of LEO satellites using global positioning system measurements," GPS Solutions, vol. 12, no. 3, pp. 187-198, 2008.

[4] B. Maurizio, L. J. John, S. J. Peter, and T. Denver, "Advanced stellar compass: onboard autonomous orbit determination, preliminary performance," Annals of the New York Academy of Sciences, vol. 1017, pp. 393-407, 2004. 
[5] D.-J. Lee and K. T. Alfriend, "Sigma point filters for efficient orbit estimation," in Proceedings of the AAS/AIAA Astrodynamics Conference, vol. 116, pp. 349-372, Advances in the Astronautical Sciences, Big Sky, Mont, USA, August 2003.

[6] P. C. P. M. Pardal, H. K. Kuga, and R. V. de Moraes, "Nonlinear sigma point Kalman filter applied to orbit determination using GPS measurement," in Proceedings of the 22nd International Meeting of the Satellite Division of The Institude of Navigation, Savannah, Ga, USA, September 2009.

[7] D.-J. Lee and T. A. Kyle, "Adaptive sigma point filtering for state and parameter estimation," in Proceedings of the AIAA/AAS Astrodynamics Specialist Conference and Exhibit, Providence, Rhode Island, USA, August 2004.

[8] S. J. Julier and J. K. Uhlmann, "A new extension of the Kalman filter to nonlinear systems," in Proceedings of the International Society for Optical Engineering (SPIE '97), vol. 3, no. 1, pp. 182-193, April 1997.

[9] S. Julier, J. Uhlmann, and H. F. Durrant-Whyte, "A new method for the nonlinear transformation of means and covariances in filters and estimators," Institute of Electrical and Electronics Engineers. Transactions on Automatic Control, vol. 45, no. 3, pp. 477-482, 2000.

[10] M. S. Arulampalam, S. Maskell, N. Gordon, and T. Clapp, “A tutorial on particle filters for online nonlinear/non-Gaussian Bayesian tracking," IEEE Transactions on Signal Processing, vol. 50, no. 2, pp. 174-188, 2002.

[11] D. Watzenig, M. Brandner, and G. Steiner, "A particle filter approach for tomographic imaging based on different state-space representations," Measurement Science and Technology, vol. 18, no. 1, pp. 30-40, 2007.

[12] R. van der Merwe, A. Doucet, N. de Freitas, and E. Wan, “The unscented particle filter," Tech. Rep. CUED/F-INFENG/TR 380, Cambridge University Engineering Department, Cambridge, UK, 2000.

[13] O. Payne and A. Marrs, "An unscented particle filter for GMTI tracking," in Proceedings of the IEEE Aerospace Conference, vol. 3, pp. 1869-1875, March 2004.

[14] M. L. Psiaki, L. Huang, and S. M. Fox, “Ground tests of magnetometer-based autonomous navigation (MAGNAV) for low-earth-orbiting spacecraft," Journal of Guidance, Control, and Dynamics, vol. 16, no. 1, pp. 206-214, 1993.

[15] H. Jung and M. L. Psiaki, "Tests of magnetometer-sun-sensor orbit determination using flight data," in Proceedings of the AIAA Guidance, Navigation, and Control Conference and Exhibit, Montreal, Canada, August 2001.

[16] C. J. Gramling and A. C. Long, "Autonomous navigation using the TDRSS Onboard Navigation System (TONS)," Advances in Space Research, vol. 16, no. 12, pp. 77-80, 1995.

[17] A. C. Long, D. Leung, D. Folta, and C. Gramling, "Autonomous navigation of high-earth satellites using celestial objects and Doppler measurements," in Proceedings of the AIAA/AAS Astrodynamics Specialist Conference, Denver, Colo, USA, August 2000.

[18] G. M. Levine, "A method of orbital navigation using optical sightings to unknown landmarks," AIAA Journal, vol. 4, no. 11, pp. 1928-1931, 1966.

[19] W. L. Brogan and J. L. Lemay, "Orbit navigation with known landmark tracking," in Proceedings of the Joint Automatic Control Conference of American Automatic Control Council, Institute of Electrical and Electronics Engineers, New York, NY, USA, 1968.

[20] S. I. Sheikh, The use of variable celestial X-Ray sources for spacecraft navigation, Ph.D. thesis, University of Maryland, College Park, Maryland, 2005.

[21] A. A. Emadzadeh and J. E. Speyer, Navigation in Space by X-Ray Pulsar, Springer, New York, NY, USA, 2011.

[22] G. Welch and G. Bishop, "An introduction to the Kalman filter," Tech. Rep. TR95-041, University of North Carolina, Chapel Hill, NC, USA, 1995.

[23] E. A. Wan and R. van der Merwe, "The unscented Kalman filter for nonlinear estimation," in Proceedings of the IEEE Adaptive Systems for Signal Processing, Communications, and Control Symposium, pp. 153-158, 2000.

[24] S. J. Julier and J. K. Uhlmann, "A general method for approximating nonlinear transformations of probability distributions," Tech. Rep. RRG, Department of Engineering Science, University of Oxford, 1996.

[25] D. A. Vallado, Fundamentals of Astrodynamics and Applications, Springer, New York, NY, USA, 3rd edition, 2007.

[26] R. H. Battin, An Introduction to the Mathematics and Methods of Astrodynamics, American Institute Aeronautics and Astronautics, New York, NY, USA, 1999. 
[27] X. L. Ning and J. C. Fang, "An autonomous celestial navigation method for LEO satellite based on unscented Kalman filter and information fusion," Aerospace Science and Technology, vol. 11, no. 2-3, pp. 222-228, 2007.

[28] I. A. O. Geomagnetism, W. G. V. P. Aeronomy, C. C. Finlay et al., "International Geomagnetic Reference Field: the eleventh generation," Geophysical Journal International, vol. 183, no. 3, pp. 1216-1230, 2010.

[29] S. Mclean, S. Macmillan, and S. Maus, "The US/UK World Magnetic Model for 2005-2010," Tech. Rep. NESDIS/NGDC-2, NOAA, 2005.

[30] Satellite Tools Kit Suite Version 8.1.1 Help System, Analytical Graphics, New York, NY, USA, 2007.

[31] The Hipparcos and Tycho Catalogues (ESA SP-1200) ESA, Noordwijk, The Netherlands, 1997.

[32] M. N. Filipski and E. J. Abdullah, "Nanosatellite navigation with the WMM2005 geomagnetic field model," Turkish Journal of Engineering and Environmental Sciences, vol. 30, no. 1, pp. 43-55, 2006.

[33] S. P. Mertikas, “Error distributions and accuracy measures in navigation: an overview," Tech. Rep. 113, 1985. 


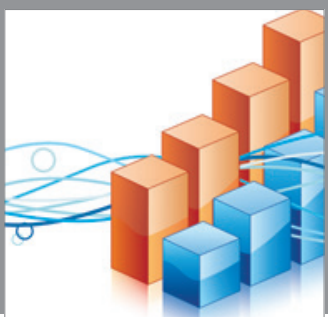

Advances in

Operations Research

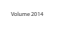

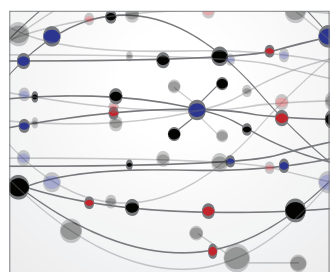

\section{The Scientific} World Journal
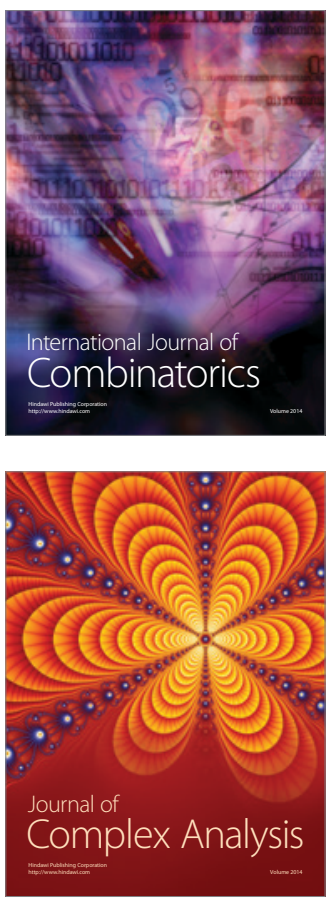

International Journal of

Mathematics and

Mathematical

Sciences
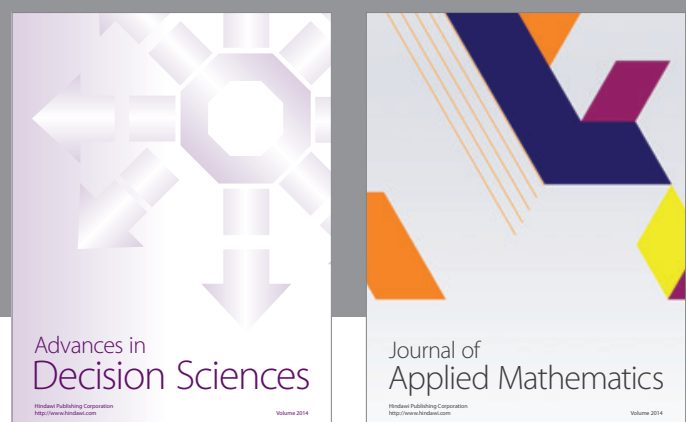

Journal of

Applied Mathematics
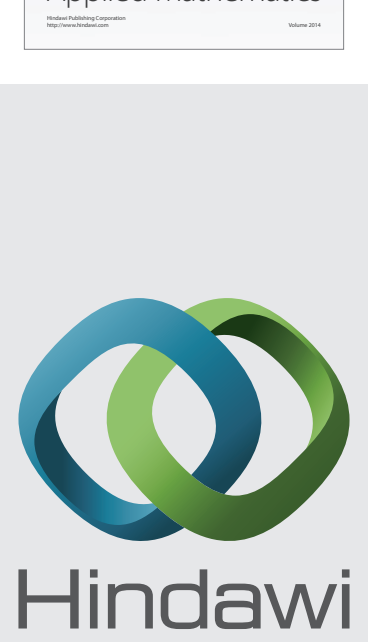

Submit your manuscripts at http://www.hindawi.com
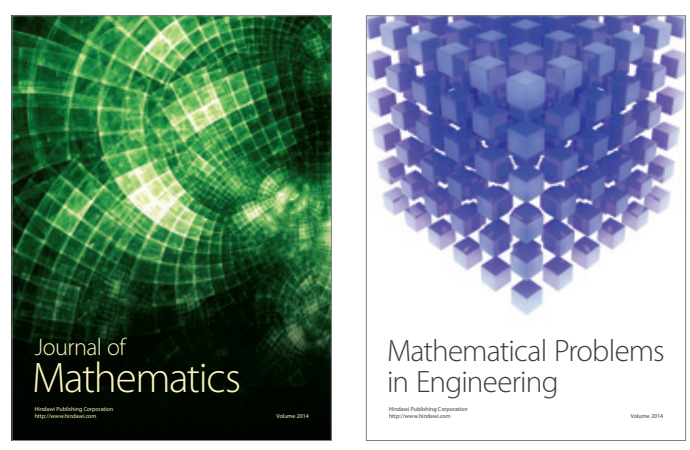

Mathematical Problems in Engineering
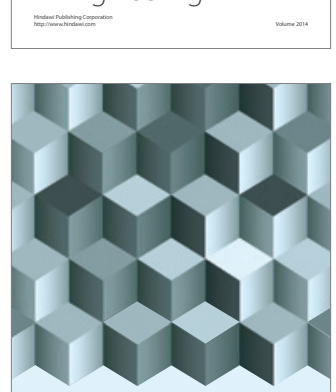

Journal of

Function Spaces
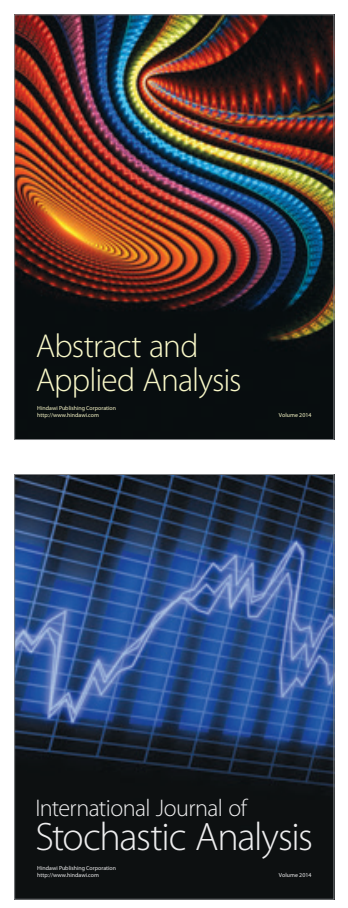

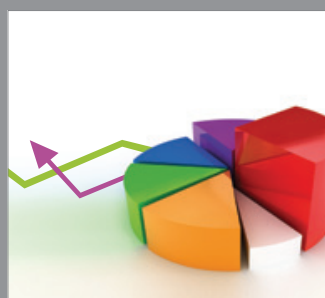

ournal of

Probability and Statistics

Promensencen
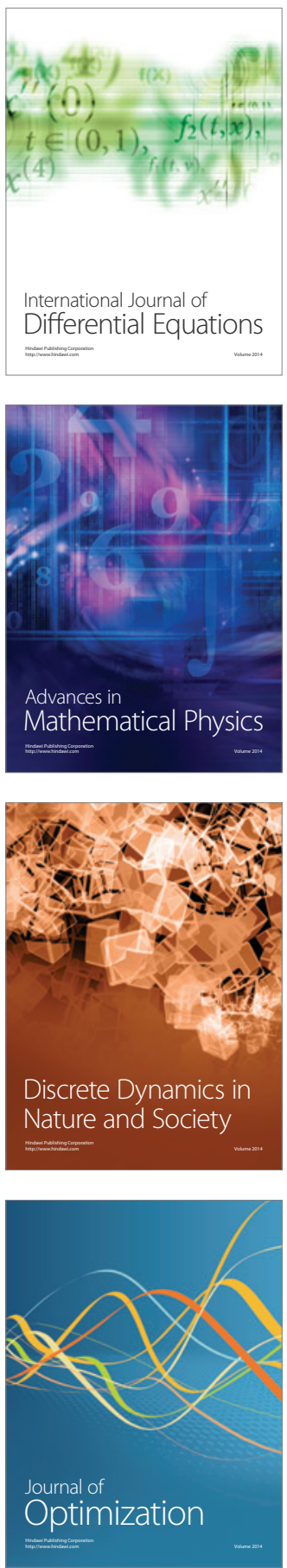